\title{
On the Essential Spectrum of Certain Non-Commutative Oscillators
}

\section{Alberto Parmeggiani}

Alberto Venni 


\title{
ON THE ESSENTIAL SPECTRUM OF CERTAIN NON-COMMUTATIVE OSCILLATORS
}

\author{
ALBERTO PARMEGGIANI AND ALBERTO VENNI
}

\begin{abstract}
We show here that the spectrum of the family of noncommutative harmonic oscillators $Q_{(\alpha, \beta)}^{\mathrm{w}}(x, D)$ for $\alpha, \beta \in \mathbb{R}_{+}$in the range $\alpha \beta=1$, is $[0,+\infty)$ and is entirely essential spectrum. The previous existing results concern the case $\alpha \beta>1$ (case in which $Q_{(\alpha, \beta)}^{\mathrm{w}}(x, D)$ is globally elliptic with a discrete spectrum whose qualitative properties are being extensively studied), and ours therefore extends the picture to the range of parameters $\alpha \beta \geq 1$.
\end{abstract}

\section{INTRODUCTION}

In this paper, we consider the following system

$$
Q^{\mathrm{w}}(x, D)=Q_{(\alpha, \beta)}^{\mathrm{w}}(x, D)=A\left(-\frac{\partial_{x}^{2}}{2}+\frac{x^{2}}{2}\right)+J\left(x \partial_{x}+\frac{1}{2}\right), \quad x \in \mathbb{R},
$$

where

$$
A=\left[\begin{array}{cc}
\alpha & 0 \\
0 & \beta
\end{array}\right], \quad J=\left[\begin{array}{cc}
0 & -1 \\
1 & 0
\end{array}\right], \alpha, \beta \in \mathbb{R}_{+}=(0,+\infty) .
$$

Put $p_{0}(x, \xi)=\left(x^{2}+\xi^{2}\right) / 2$. System (1.1) is then the Weyl quantization of the matrix-valued quadratic form on $T^{*} \mathbb{R}=\mathbb{R} \times \mathbb{R}$

$$
Q(x, \xi)=Q_{(\alpha, \beta)}(x, \xi)=A p_{0}(x, \xi)+i J x \xi .
$$

Clearly, one has $Q(x, \xi)^{*}=Q(x, \xi)$ for all $(x, \xi) \in \mathbb{R} \times \mathbb{R}$.

The operator $Q^{\mathrm{w}}(x, D)$ shall be throughout realized as an unbounded operator $\mathrm{Q}$ in $L^{2}\left(\mathbb{R} ; \mathbb{C}^{2}\right)$ with maximal domain

$$
\mathrm{D}=\left\{u \in L^{2}\left(\mathbb{R} ; \mathbb{C}^{2}\right) ; Q^{\mathrm{w}}(x, D) u \in L^{2}\left(\mathbb{R} ; \mathbb{C}^{2}\right) \text { (in the } \mathscr{S}^{\prime} \text {-sense) }\right\},
$$

i.e. $\mathrm{Q}$ is the maximal realization of $Q^{\mathrm{w}}(x, D)$.

2010 Mathematics Subject Classification. Primary 35P05; Secondary 34L40, 47G30, 47N50.

Key words and phrases: non-commutative harmonic oscillator; essential spectrum.

The first author wishes to thank the Erwin Schrödinger International Institute for Mathematical Physics, where the final version of this paper was prepared, for the wonderful hospitality, and the organizers of the program held there, H. G. Feichtinger, C. Gröchenig, M. de Hoop and M. Ruzhansky. 
When $\alpha \beta>1$ one has $\operatorname{det} Q(x, \xi) \approx\left(x^{2}+\xi^{2}\right)^{2}$, whence it follows that $Q^{\mathrm{w}}$ is a (classical) globally elliptic self-adjoint operator in $L^{2}\left(\mathbb{R} ; \mathbb{C}^{2}\right)$ (see [20]), whence its realization $\mathrm{Q}$ has domain

$$
B^{2}\left(\mathbb{R} ; \mathbb{C}^{2}\right)=\left\{u \in L^{2}\left(\mathbb{R} ; \mathbb{C}^{2}\right) ; \sum_{j, k \geq 0, j+k \leq 2}\left\|x^{j} \partial_{x}^{k} u\right\|_{L^{2}\left(\mathbb{R} ; \mathbb{C}^{2}\right)}<+\infty\right\},
$$

and is self-adjoint. Since $B^{2}\left(\mathbb{R} ; \mathbb{C}^{2}\right)$ is compactly embedded into $L^{2}\left(\mathbb{R} ; \mathbb{C}^{2}\right)$, we have that the spectrum of $Q$ is discrete, made of a diverging (to $+\infty$ ) sequence of real eigenvalues with finite multiplicities, and it turns out (see [14]) that its lowest eigenvalue is positive. Hence (with repetitions according to the multiplicity)

$$
\operatorname{Spec}(Q)=\left\{0<\lambda_{1} \leq \lambda_{2} \leq \ldots \rightarrow+\infty\right\} .
$$

System (1.1) is called non-commutative harmonic oscillator (NCHO for short), in the terminology introduced by Wakayama and the first author in [13] and [14], and (1.1) is actually a normal form of the class introduced there (see [14]).

Wakayama and the first author gave in $[13,14]$, a qualitative description of the spectrum of $Q^{\mathrm{W}}$ when $\alpha \beta>1$, by using $\mathfrak{s l}_{2}(\mathbb{R})$-symmetries to construct suitable creation-annihilation operators and a basis B made of "twisted" vector-valued Hermite functions.

The case $\alpha=\beta$ is completely understood: the system is unitarily equivalent (through automorphisms of $\mathscr{S}\left(\mathbb{R} ; \mathbb{C}^{2}\right)$ and $\mathscr{S}^{\prime}\left(\mathbb{R} ; \mathbb{C}^{2}\right)$ ), according to the cases $\alpha=\beta>1, \alpha=\beta=1$ and $0<\alpha=\beta<1$, respectively, to the scalar harmonic oscillator $\sqrt{\alpha^{2}-1}\left(-\partial_{x}^{2}+x^{2}\right) / 2$, to the scalar $-\partial_{x}^{2} / 2$, to the scalar $\sqrt{1-\alpha^{2}}\left(-\partial_{x}^{2}-x^{2}\right) / 2$, respectively (see Corollary 4.1 of [14]), and one has the following result.

Theorem 1.1 ( [14]). When $\alpha=\beta>1$ one has

$$
\operatorname{Spec}(\mathrm{Q})=\left\{\sqrt{\alpha^{2}-1}\left(N+\frac{1}{2}\right) ; N \in \mathbb{Z}_{+}\right\}
$$

(where $\mathbb{Z}_{+}=\{0,1, \ldots\}$ ), with eigenvalues of multiplicity 2 .

When $\alpha=\beta=1$ one has

$$
\operatorname{Spec}(\mathrm{Q})=\operatorname{Spec}_{\mathrm{ess}}(\mathrm{Q})=[0,+\infty)
$$

When $\alpha=\beta<1$ one has

$$
\operatorname{Spec}(\mathrm{Q})=\operatorname{Spec}_{\mathrm{ess}}(\mathrm{Q})=\mathbb{R} .
$$

Here $\mathrm{Spec}_{\mathrm{ess}}$ denotes the essential spectrum (the complement in $\mathbb{C}$ of the discrete spectrum).

It is interesting to notice the appearance of the "symplectic" parameter $\sqrt{\alpha^{2}-1}$ (denoted by $\ell=\sqrt{\alpha \beta-1}$ in $[13,14]$ ).

When $\alpha \neq \beta$ things are highly nontrivial. In this case, when $\alpha \beta>1$, in [14] (see also [13]) to understand the spectrum two kinds of sets, $\Sigma_{0}^{ \pm}$and $\Sigma_{\infty}^{ \pm}$, were introduced. (The \pm stands in this case for the parity: the system 
preserves parity, whence it follows that one can study, separately, the even case, + , and the odd one, - , respectively.) The sets $\Sigma_{0}^{ \pm}$are described as the sets of those eigenvalues that are roots of particular polynomials, whereas the sets $\Sigma_{\infty}^{ \pm}$are described as the sets of those eigenvalues that are zeroes of particular meromorphic functions (defined through continued fractions). These polynomials and meromorphic functions are related to certain threeterm recurrence systems. Corresponding to eigenvalues belonging to $\Sigma_{0}=$ $\Sigma_{0}^{+} \cup \Sigma_{0}^{-}$one has eigenfunctions which are written as a linear combination of finitely many elements of the basis $\mathrm{B}$, whereas corresponding to eigenvalues belonging to $\Sigma_{\infty}=\Sigma_{\infty}^{+} \cup \Sigma_{\infty}^{-}$one has eigenfunctions which are written as a linear combination of infinitely many elements of the basis B. While one always has $\Sigma_{0}^{+} \cap \Sigma_{0}^{-}=\emptyset$, the intersection $\Sigma_{\infty}^{+} \cap \Sigma_{\infty}^{-}$has a very difficult description and is yet to be understood. Upon defining

$$
E_{ \pm}(\lambda)=\left\{u \in L^{2}\left(\mathbb{R} ; \mathbb{C}^{2}\right) ; Q^{\mathrm{w}}(x, D) u=\lambda u, u \text { even } / \text { odd }\right\},
$$

one has the following theorem.

Theorem 1.2 ( [14]; see also [13]). When $\alpha \neq \beta$, one has

$$
\begin{gathered}
\Sigma_{0}^{+} \subset \Sigma_{\infty}^{+}, \Sigma_{0}^{-} \subset \Sigma_{\infty}^{-}, \\
\operatorname{Spec}\left(Q^{\mathrm{w}}(x, D)\right)=\Sigma_{\infty}^{+} \cup \Sigma_{\infty}^{-},
\end{gathered}
$$

and

$$
\operatorname{dim} E_{ \pm}(\lambda)=\left\{\begin{array}{l}
2, \quad \text { whenever } \lambda \in \Sigma_{0}^{ \pm} \\
1, \quad \text { whenever } \lambda \in \Sigma_{\infty}^{ \pm} \backslash \Sigma_{0}^{ \pm}
\end{array}, \pm-\right. \text {respectively }
$$

Notice that the theorem says nothing about whether $\Sigma_{0}^{ \pm} \cap\left(\Sigma_{\infty}^{\mp} \backslash \Sigma_{0}^{\mp}\right) \neq \emptyset$, case that would yield an eigenvalue of multiplicity 3 .

The sets $\Sigma_{0}^{ \pm}$and $\Sigma_{\infty}^{ \pm}$, although explicitly described, are complicated (see $[13,14]$ and also $[15,17,18])$, and it would be desirable to have also other (hopefully simpler) descriptions.

It is a remarkable fact, proved by Ochiai in [11] (see also [12]), that the spectral problem for $Q^{\mathrm{w}}$ is equivalent to a family of third-order Fuchsian differential equations with four regular singularities in the complex unit disk. Furthermore, important work on the spectral zeta-function $\zeta_{Q}(s)$, and its special values, associated with $Q^{\mathrm{w}}(x, D)$, defined by

$$
\zeta_{\mathrm{Q}}(s)=\sum_{\lambda \in \operatorname{Spec}(\mathrm{Q})} \frac{1}{\lambda^{s}}, \quad s \in \mathbb{C}, \operatorname{Re} s>1,
$$

has been started by Ichinose and Wakayama (see [6,7]; see also [18] and [8]). It is also worth mentioning that numerical study of the spectrum $Q^{\mathrm{w}}(x, D)$ has been carried out by Nagatou, Nakao and Wakayama in [10], and that one can study the spectrum by Rellich's perturbation theory in the limit $\alpha \beta \rightarrow+\infty$ with $\alpha / \beta$ a fixed constant $\neq 1$ (see [15]). Furthermore, the study of Poisson-type relations for the spectral distribution, and clustering theorems of the spectrum were proved in Parmeggiani $[16,17]$ (see also [18]). 
As for the multiplicity of the lowest eigenvalue, one has results in Parmeggiani [15], in Hiroshima and Sasaki [3], and in the more recent paper by Wakayama [21]; however, our knowledge of the lowest eigenvalue is still incomplete. It is finally worth mentioning the recent study of Dicke-type crossings among the eigenvalues of certain families of NCHOs carried out by Hirokawa in [2], which is related to the study of self-adjoint operators with non-commutative coefficients such as the Rabi model or the JaynesCumming model, describing the interaction between a one-mode photon and a two-level atom.

In this paper, we extend the knowledge of the spectrum of $Q_{(\alpha, \beta)}^{\mathrm{w}}(x, D)$, thought of as its maximal realization $\mathrm{Q}$, to the case $\alpha \beta=1$, with $\alpha, \beta>0$, case in which the $\mathrm{NCHO}$ is no longer globally elliptic, proving that when $\alpha \beta=1$ one has

$$
\operatorname{Spec}(Q)=\operatorname{Spec}_{\text {ess }}(Q)=[0,+\infty) .
$$

The proof is based on a metaplectic factorization (in $\mathscr{S}^{\prime}\left(\mathbb{R} ; \mathbb{C}^{2}\right)$ ) of $Q^{\mathrm{w}}(x, D)$, with local metaplectic operators (i.e. none of the metaplectic operators involved is the Fourier transform).

\section{A fundamental Lemma And a FeW CONSEquences of it}

We prove in this section a fundamental lemma, following the approach of [18] in his proof of Theorem 8.2.1. Although the lemma will be used only in the case $\alpha \beta=1$, we shall prove it in the general case (i.e. $\alpha, \beta>0$ and no restriction on $\alpha \beta$ ) because it will be useful also in a subsequent study.

We have to introduce some notation. For $\alpha, \beta \in \mathbb{R}_{+}$, let

$$
0<\delta:=\sqrt{\alpha \beta}, \mathbf{s}(\delta):=\left\{\begin{array}{l}
\operatorname{sgn}(\alpha \beta-1), \delta \neq 1, \\
0, \alpha \beta=1 .
\end{array}, \epsilon:=\sqrt{|\alpha \beta-1|},\right.
$$

and $\omega_{ \pm}:=\frac{\alpha \pm \beta}{2}$. Let $v_{ \pm}:=\frac{1}{\sqrt{2}}\left[\begin{array}{c}1 \\ \mp i\end{array}\right]$ be the orthonormal eigenvectors of $J$ belonging to $\pm i$, respectively. Hence $J v_{ \pm}= \pm i v_{ \pm}$and, furthermore, $K J v_{ \pm}=v_{\mp}$, where $K=\left[\begin{array}{ll}0 & 1 \\ 1 & 0\end{array}\right]$. Let $W_{0}:=\left[v_{+} \mid v_{-}\right]$. Let us consider the (global, in the sense of Shubin, see [20], or [9], or [18]) symbols

$$
\begin{gathered}
p_{0}(x, \xi)=\frac{\xi^{2}+x^{2}}{2}, e(x, \xi)=x \xi \\
p_{\delta}(x, \xi)=\frac{\xi^{2}+\mathrm{s}(\delta) x^{2}}{2}, L_{\delta}(x, \xi)=\frac{\xi^{2}+\left(\delta^{2}-1\right) x^{2}}{2},
\end{gathered}
$$

and the linear symplectomorphisms $\chi_{\delta}, \chi_{ \pm}: \mathbb{R} \times \mathbb{R} \longrightarrow \mathbb{R} \times \mathbb{R}$, where

$$
\chi_{\delta}:(x, \xi) \longmapsto\left(\delta^{1 / 2} x, \delta^{-1 / 2} \xi\right), \quad \chi_{ \pm}:(x, \xi) \longmapsto(x, \xi \pm x),
$$

which are associated with the metaplectic operators

$$
U_{\delta}: f(x) \longmapsto\left(U_{\delta} f\right)(x)=\delta^{-1 / 4} f\left(x / \delta^{1 / 2}\right),
$$




$$
U_{ \pm}: f(x) \longmapsto\left(U_{ \pm} f\right)(x)=e^{ \pm i x^{2} / 2} f(x)
$$

respectively. Notice that $U_{-}^{*}=U_{-}^{-1}=U_{+}$, and that $U_{\delta}^{*}=U_{\delta}^{-1}=U_{1 / \delta}$.

Lemma 2.1. There exist metaplectic operators $\mathrm{U}_{0}$ and $\mathrm{U}_{\epsilon}$, isometries of $L^{2}\left(\mathbb{R} ; \mathbb{C}^{2}\right)$ and automorphisms of $\mathscr{S}^{\prime}\left(\mathbb{R} ; \mathbb{C}^{2}\right)$ and of $\mathscr{S}\left(\mathbb{R} ; \mathbb{C}^{2}\right)$, such that for all $\alpha, \beta \in \mathbb{R}_{+}$and for all $\lambda \in \mathbb{C}$, on $\mathscr{S}^{\prime}\left(\mathbb{R} ; \mathbb{C}^{2}\right)$ the operator $Q^{\mathrm{w}}(x, D)-\lambda$ can be factored as (recall that $D_{x}=-i \partial_{x}$ )

$$
Q^{\mathrm{w}}(x, D)-\lambda=A^{1 / 2} \mathrm{U}_{0}^{*}\left(\frac{1}{2} D_{x}^{2}-\lambda V(x)\right) \mathrm{U}_{0} A^{1 / 2}, \text { when } \epsilon=0,
$$

and as

(2.2) $Q^{\mathrm{w}}(x, D)-\lambda=\frac{1}{\delta} A^{1 / 2} \mathrm{U}_{\epsilon}^{*}\left(\epsilon p_{\delta}^{\mathrm{w}}(x, D)-\frac{\lambda}{\delta} V_{\epsilon}(x)\right) \mathrm{U}_{\epsilon} A^{1 / 2}$, when $\epsilon>0$, where

$$
V(x)=\left[\begin{array}{cc}
\omega_{+} & -\omega_{-} e^{-i x^{2}} \\
-\omega_{-} e^{i x^{2}} & \omega_{+}
\end{array}\right], \quad V_{\epsilon}(x)=V\left(x / \epsilon^{1 / 2}\right)
$$

and

$$
\mathrm{U}_{0}=\frac{1}{\sqrt{2}}\left[\begin{array}{cc}
U_{-} U_{\delta}^{*} & 0 \\
0 & U_{+} U_{\delta}^{*}
\end{array}\right]\left[\begin{array}{cc}
1 & i \\
1 & -i
\end{array}\right], \quad \mathrm{U}_{\epsilon}=\left(U_{1 / \epsilon}^{*} \otimes I_{2}\right) \mathrm{U}_{0} .
$$

The metaplectic operators $\mathrm{U}_{0}$ and $\mathrm{U}_{\epsilon}$ are local (i.e. leave $C_{0}^{\infty}\left(\mathbb{R} ; \mathbb{C}^{2}\right.$ ) invariant).

Proof. One writes

$$
Q^{\mathrm{w}}(x, D)-\lambda=\frac{1}{\delta} A^{1 / 2}\left(\delta p_{0}^{\mathrm{w}}(x, D)+i J e^{\mathrm{w}}(x, D)-\lambda \delta A^{-1}\right) A^{1 / 2}
$$

Hence, thinking of $A^{1 / 2}$ as an automorphism of $\mathscr{S}^{\prime}\left(\mathbb{R} ; \mathbb{C}^{2}\right)$, we have that $\left(Q^{\mathrm{w}}(x, D)-\lambda\right) u=0$ in $\mathscr{S}^{\prime}\left(\mathbb{R} ; \mathbb{C}^{2}\right)$ is equivalent to

$$
\left(\delta p_{0}^{\mathrm{w}}(x, D)+i J e^{\mathrm{w}}(x, D)-\lambda \delta A^{-1}\right) v=0, \quad v:=A^{1 / 2} u \in \mathscr{S}^{\prime}\left(\mathbb{R} ; \mathbb{C}^{2}\right) .
$$

Since

we have

$$
\delta p_{0}(x, \xi) \mp x \xi=\left(L_{\delta} \circ \chi_{\mp} \circ \chi_{\delta}^{-1}\right)(x, \xi),
$$

$$
\delta p_{0}^{\mathrm{w}}(x, D) \mp e^{\mathrm{w}}(x, D)=U_{\delta} U_{\mp}^{-1} L_{\delta}^{\mathrm{w}}(x, D) U_{\mp} U_{\delta}^{-1} .
$$

As $A^{-1}=\left(\omega_{+} I-\omega_{-} K J\right) / \delta^{2}$, we may write, using the diagonalizer $W_{0}$ of $J$,

$$
\begin{gathered}
W_{0}^{*}\left(\delta p_{0}^{\mathrm{w}}(x, D)+i J e^{\mathrm{w}}(x, D)-\lambda \delta A^{-1}\right) W_{0}= \\
=\left[\begin{array}{cc}
\delta p_{0}^{\mathrm{w}}(x, D)-e^{\mathrm{w}}(x, D)-\frac{\lambda}{\delta} \omega_{+} & \frac{\lambda}{\delta} \omega_{-} \\
\frac{\lambda}{\delta} \omega_{-} & \delta p_{0}^{\mathrm{w}}(x, D)+e^{\mathrm{w}}(x, D)-\frac{\lambda}{\delta} \omega_{+}
\end{array}\right]= \\
=\underbrace{\left[\begin{array}{cc}
U_{\delta} U_{+} & 0 \\
0 & U_{\delta} U_{-}
\end{array}\right]}_{=: \mathrm{U}^{*}} L_{\delta}^{\mathrm{w}}(x, D) \underbrace{\left[\begin{array}{cc}
U_{-} U_{\delta}^{*} & 0 \\
0 & U_{+} U_{\delta}^{*}
\end{array}\right]}_{=: \mathrm{U}}-\frac{\lambda}{\delta}\left[\begin{array}{cc}
\omega_{+} & -\omega_{-} \\
-\omega_{-} & \omega_{+}
\end{array}\right]= \\
=\mathrm{U}^{*}\left(L_{\delta}^{\mathrm{w}}(x, D)-\frac{\lambda}{\delta} \mathrm{U}\left[\begin{array}{cc}
\omega_{+} & -\omega_{-} \\
-\omega_{-} & \omega_{+}
\end{array}\right] \mathrm{U}^{*}\right) \mathrm{U}=
\end{gathered}
$$


(since $U_{+} U_{\delta}^{*}\left(U_{-} U_{\delta}^{*}\right)^{*}: f \longmapsto e^{i x^{2}} f$ )

$$
=\mathrm{U}^{*}\left(L_{\delta}^{\mathrm{w}}(x, D)-\frac{\lambda}{\delta} V(x)\right) \mathrm{U},
$$

where

$$
V(x):=\left[\begin{array}{cc}
\omega_{+} & -\omega_{-} e^{-i x^{2}} \\
-\omega_{-} e^{i x^{2}} & \omega_{+}
\end{array}\right] .
$$

Hence, at this point we have obtained the factorization

$$
Q^{\mathrm{w}}(x, D)-\lambda=\frac{1}{\delta} A^{1 / 2} W_{0} \mathrm{U}^{*}\left(L_{\delta}^{\mathrm{w}}(x, D)-\frac{\lambda}{\delta} V(x)\right) \mathrm{U} W_{0}^{*} A^{1 / 2} .
$$

Now, when $\epsilon=0$, that is $\alpha \beta=1=\delta$, we then have $L_{1}^{\mathrm{w}}(x, D)=D_{x}^{2} / 2$, so that

where

$$
Q^{\mathrm{w}}(x, D)-\lambda=A^{1 / 2} \mathrm{U}_{0}^{*}\left(\frac{1}{2} D_{x}^{2}-\lambda V(x)\right) \mathrm{U}_{0} A^{1 / 2},
$$

$$
\mathrm{U}_{0}=\mathrm{U} W_{0}^{*}=\frac{1}{\sqrt{2}}\left[\begin{array}{cc}
U_{-} U_{\delta}^{*} & 0 \\
0 & U_{+} U_{\delta}^{*}
\end{array}\right]\left[\begin{array}{cc}
1 & i \\
1 & -i
\end{array}\right]
$$

which is the sought factorization, as claimed.

Next, when $\epsilon>0$, consider $\chi_{1 / \epsilon}:(x, \xi) \longmapsto\left(\epsilon^{-1 / 2} x, \epsilon^{1 / 2} \xi\right)$, and the associated metaplectic operator $U_{1 / \epsilon}: f \longmapsto \epsilon^{1 / 4} f\left(\epsilon^{1 / 2} \cdot\right)$. Since

$$
U_{1 / \epsilon}^{*} L_{\delta}^{\mathrm{w}}(x, D) U_{1 / \epsilon}=\left(L_{\delta} \circ \chi_{1 / \epsilon}\right)^{\mathrm{w}}(x, D)=\frac{\epsilon}{2}\left(D_{x}^{2}+\mathrm{s}(\delta) x^{2}\right)=\epsilon p_{\delta}^{\mathrm{w}}(x, D),
$$

we get from $(2.3)$

$$
Q^{\mathrm{w}}(x, D)-\lambda=\frac{1}{\delta} A^{1 / 2} \mathrm{U}_{\epsilon}^{*}\left(\epsilon p_{\delta}^{\mathrm{w}}(x, D)-\frac{\lambda}{\delta} V_{\epsilon}(x)\right) \mathrm{U}_{\epsilon} A^{1 / 2},
$$

where this time

$$
\mathrm{U}_{\epsilon}=\left(U_{1 / \epsilon}^{*} \otimes I_{2}\right) \mathrm{U} W_{0}^{*}
$$

and

$$
V_{\epsilon}(x)=U_{1 / \epsilon}^{*} V(x) U_{1 / \epsilon}=V\left(x / \epsilon^{1 / 2}\right)
$$

(of course, as a multiplication operator). This concludes the proof of the lemma.

Therefore we have in particular that

$$
Q^{\mathrm{w}}(x, D)=\frac{\epsilon}{\delta} A^{1 / 2} \mathrm{U}_{\epsilon}^{*} p_{\delta}^{\mathrm{w}}(x, D) \mathrm{U}_{\epsilon} A^{1 / 2}, \text { when } \epsilon>0,
$$

and

$$
Q^{\mathrm{w}}(x, D)=\frac{1}{2} A^{1 / 2} \mathrm{U}_{0}^{*} D_{x}^{2} \mathrm{U}_{0} A^{1 / 2}, \text { when } \epsilon=0,
$$

where the metaplectic operators $\mathrm{U}_{0}$ and $\mathrm{U}_{\epsilon}$ are given in (2.4) and (2.5), respectively.

Notice that neither $\mathrm{U}_{0}$ nor $\mathrm{U}_{\epsilon}$ commutes with $A$. 
Recalling that $Q^{\mathrm{w}}(x, D)$ is realized as the maximal operator $\mathrm{Q}$, with domain

$$
\mathrm{D}=\mathrm{D}_{\alpha, \beta}=\left\{u \in L^{2}\left(\mathbb{R} ; \mathbb{C}^{2}\right) ; Q^{\mathrm{w}}(x, D) u \in L^{2}\left(\mathbb{R} ; \mathbb{C}^{2}\right)\right\},
$$

we have that, when $\alpha \beta=1$, D can be described as

$$
\begin{array}{r}
\mathrm{D}=\left\{u \in L^{2}\left(\mathbb{R} ; \mathbb{C}^{2}\right) ; D_{x}^{2} \mathrm{U}_{0} A^{1 / 2} u \in L^{2}\left(\mathbb{R} ; \mathbb{C}^{2}\right)\right\}= \\
=\left\{u \in L^{2}\left(\mathbb{R} ; \mathbb{C}^{2}\right) ; \mathrm{U}_{0} A^{1 / 2} u \in H^{2}\left(\mathbb{R} ; \mathbb{C}^{2}\right)\right\},
\end{array}
$$

whereas, when $0<\alpha \beta<1$, it can be described as

$$
\mathrm{D}=\left\{u \in L^{2}\left(\mathbb{R} ; \mathbb{C}^{2}\right) ;\left(D_{x}^{2}-x^{2}\right) \mathrm{U}_{\epsilon} A^{1 / 2} u \in L^{2}\left(\mathbb{R} ; \mathbb{C}^{2}\right)\right\}
$$

Moreover, when $\alpha \beta \geq 1$ we have $Q^{\mathrm{w}}(x, D) \geq 0$ on $\mathscr{S}\left(\mathbb{R} ; \mathbb{C}^{2}\right)$. This is already well-known when $\alpha \beta>1$ (see [14], or [18]), and when $\alpha, \beta \in \mathbb{R}_{+}$with $\alpha \beta=1$ it follows from Lemma 2.1, for we have

$$
\begin{aligned}
\left(Q^{\mathrm{w}}(x, D) u, u\right)=\frac{1}{2} & \left(D_{x}^{2} \mathrm{U}_{0} A^{1 / 2} u, \mathrm{U}_{0} A^{1 / 2} u\right)= \\
& =\frac{1}{2}\left\|\partial_{x} \mathrm{U}_{0} A^{1 / 2} u\right\|_{L^{2}}^{2} \geq 0, \quad \forall u \in \mathscr{S}\left(\mathbb{R} ; \mathbb{C}^{2}\right) .
\end{aligned}
$$

As another immediate consequence of Lemma 2.1, we may establish the self-adjointness of $\mathrm{Q}$, also for the range of values $0<\alpha \beta \leq 1$.

This also follows from the arguments of Hörmander [5], whose extension to our system presents no problem: using the Weyl-Hörmander pseudodifferential calculus in the "global" setting (see, e.g., [20], or [9], or [18]) one sees that $\mathrm{Q}$ is the closure of its restriction of $\mathscr{S}\left(\mathbb{R} ; \mathbb{C}^{2}\right)$ on which it is symmetric.

However, we shall here prove directly the self-adjointness of $Q$ for the sake of having a self-contained approach.

Recall also that the operator $D_{x}^{2}-x^{2}$, realized as an unbounded operator in $L^{2}$ defined on its maximal domain, is self-adjoint, with (essential) spectrum $(-\infty,+\infty)$.

Corollary 2.2. The operator $\mathrm{Q}$ is self-adjoint also for $0<\alpha \beta \leq 1$.

Proof. We give a proof in the case $\alpha \beta=1$, the other case $0<\alpha \beta<1$ being completely similar. For simplicity we write $\mathrm{D}_{*}$ for $\mathscr{D}\left(\mathrm{Q}^{*}\right)$. Put $\mathrm{F}=\mathrm{U}_{0} A^{1 / 2}$. Since

$$
\mathrm{D}_{*}=\left\{v \in L^{2} ; \mathrm{D}=\mathrm{F}^{-1}\left(H^{2}\right) \ni u \longmapsto(Q u, v)=\frac{1}{2}\left(D_{x}^{2} \mathrm{~F} u, \mathrm{~F} v\right) \text { is bndd }\right\},
$$

using (2.7) we may consider

$$
\mathrm{F}\left(\mathrm{D}_{*}\right)=\left\{\mathrm{F} v \in L^{2} ; H^{2} \ni \mathrm{F} u \longmapsto\left(D_{x}^{2} \mathrm{~F} u, \mathrm{~F} v\right) \text { is bndd }\right\} .
$$

As $H^{2}$ is the maximal domain of $D_{x}^{2}$, we thus conclude that $\mathrm{F}\left(\mathrm{D}_{*}\right)=$ $H^{2}\left(\mathbb{R} ; \mathbb{C}^{2}\right)$, whence $\mathrm{D}_{*}=\mathrm{D}$.

Therefore, in particular, for all $\alpha, \beta \in \mathbb{R}_{+}$with $\alpha \beta=1$, the operator $\mathrm{Q}$ is self-adjoint, with spectrum contained in $[0,+\infty)$. We know that when $\alpha=\beta=1$, our NCHO $Q^{\mathrm{w}}(x, D)$ is thus isometrically equivalent to $\left(D_{x}^{2} / 2\right) I_{2}$, whence the spectrum of $Q$ is indeed the whole half-ray $[0,+\infty)$. In the next 
section, we will see that this is indeed the case also for all $\alpha, \beta \in \mathbb{R}_{+}$with $\alpha \beta=1$.

We close the section by stating the following classical characterization of the bottom of the essential spectrum of a self-adjoint operator (Persson's Theorem; see [1]), that we state already in the case of a self-adjoint system of the kind we shall have to consider in what follows (the generalization to these systems presents no problem).

Proposition 2.3. Let $F=F^{*} \in L^{\infty}\left(\mathbb{R} ; \mathrm{M}_{2}(\mathbb{C})\right) \cap C^{\infty}\left(\mathbb{R} ; \mathrm{M}_{2}(\mathbb{C})\right)$, and let $\mathrm{P}=\mathrm{P}^{*}$ be the realization of $D_{x}^{2} / 2+F(x)$ with domain $H^{2}\left(\mathbb{R} ; \mathbb{C}^{2}\right)$ (i.e. the maximal realization). Hence $\mathbf{P}$ is semibounded from below. We have

$$
\inf \operatorname{Spec}_{\mathrm{ess}}(\mathrm{P})=\sup _{K \subset \subset \mathbb{R}} \inf \left\{\frac{(\mathrm{P} \varphi, \varphi)}{\|\varphi\|^{2}} ; \varphi \in C_{0}^{\infty}\left(\mathbb{R} \backslash K ; \mathbb{C}^{2}\right), \varphi \neq 0\right\} .
$$

\section{Proof of the Main Result}

In this section we prove our result, that we recall next.

Theorem 3.1. Let $\alpha, \beta \in \mathbb{R}_{+}$with $\alpha \beta=1$. For $Q^{\mathrm{w}}(x, D)$, realized as the maximal extension $\mathrm{Q}$ with domain $\mathrm{D}$ (see (2.8)), we have

$$
\operatorname{Spec}(\mathrm{Q})=\operatorname{Spec}_{\mathrm{ess}}(\mathrm{Q})=[0,+\infty) \text {. }
$$

Proof. We shall follow the approach by Sasaki [19]. Since $Q$ is self-adjoint and nonnegative on $\mathrm{D}$, we shall prove that for any given $\lambda \in[0,+\infty)$, the operator $\mathrm{Q}-\lambda$ has 0 in the essential spectrum (in other words, by the Weyl criterion, see e.g. [4], for each $\lambda \geq 0$ one may construct a corresponding Weyl sequence). Therefore, by Lemma 2.1, this is in turn reduced to proving that for every fixed $\lambda \in[0,+\infty)$, the operator

$$
\frac{1}{2} D_{x}^{2}-\lambda V(x)=: \frac{1}{2} D_{x}^{2}-\lambda \omega_{+}+\lambda \omega_{-}\left[\begin{array}{cc}
0 & e^{-i x^{2}} \\
e^{i x^{2}} & 0
\end{array}\right]=: \frac{1}{2} D_{x}^{2}-\mu+F(x)
$$

has 0 in the essential spectrum. To prove this, it suffices to prove that $\operatorname{Spec}_{\text {ess }}\left(\frac{1}{2} D_{x}^{2}+F(x)\right)=[0,+\infty)$, because one may then construct a Weyl sequence for $\frac{1}{2} D_{x}^{2}+F(x)-\mu$, for all $\mu \in[0,+\infty)$. For short, we keep writing $\frac{1}{2} D_{x}^{2}+F(x)$ also for its maximal realization in $L^{2}\left(\mathbb{R} ; \mathbb{C}^{2}\right)$ with maximal domain $H^{2}\left(\mathbb{R} ; \mathbb{C}^{2}\right)$, on which it is self-adjoint, by virtue of the fact that for the vector-valued potential $F$ we have $F=F^{*} \in L^{\infty}\left(\mathbb{R} ; \mathrm{M}_{2}(\mathbb{C})\right) \cap C^{\infty}\left(\mathbb{R} ; \mathrm{M}_{2}(\mathbb{C})\right)$.

We have the following basic proposition.

Proposition 3.2. $\operatorname{Spec}_{\mathrm{ess}}\left(\frac{1}{2} D_{x}^{2}+F(x)\right)=[0,+\infty)$.

Proof of the proposition. Since (as a Riemann generalized integral) one has $\int_{0}^{\infty} e^{i x^{2}} d x=\sqrt{2 \pi}(1+i) / 4=: \omega_{0}$, we note that

$$
\lim _{R \rightarrow+\infty} \pm \int_{0}^{ \pm R} F(x) d x=\lambda \omega_{-}\left[\begin{array}{cc}
0 & \bar{\omega}_{0} \\
\omega_{0} & 0
\end{array}\right]
$$


Define, for $R \in \mathbb{R}$,

$$
W_{+}(x ; R):=\int_{R}^{x} F(s) d s, \quad W_{-}(x ; R)=\int_{x}^{-R} F(s) d s .
$$

Then

$W_{ \pm}=W_{ \pm}^{*}, \quad W_{ \pm}^{\prime}(x ; R)= \pm F(x), \quad$ and $\quad W_{ \pm}( \pm R ; R)=0 \quad$ ( \pm- respectively). By virtue of (3.10) we have that there exists $C_{0}>0$ such that for all $R$

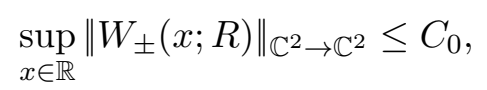

and that

$$
\alpha(R):=\sup _{x \geq R}\left\|W_{+}(\cdot, R)\right\|_{\mathbb{C}^{2} \rightarrow \mathbb{C}^{2}}+\sup _{x \leq-R}\left\|W_{-}(\cdot, R)\right\|_{\mathbb{C}^{2} \rightarrow \mathbb{C}^{2}} \longrightarrow 0, \text { as } R \rightarrow+\infty .
$$

For $u \in C_{0}^{\infty}\left(\mathbb{R} ; \mathbb{C}^{2}\right)$, and $\chi_{R}$ the characteristic function of $[-R, R]$, consider

$$
\begin{gathered}
(u, F u)=\int_{\mathbb{R}}\langle u(x), F(x) u(x)\rangle_{\mathbb{C}^{2}} d x=\left(u, \chi_{R} F u\right)+\left(u,\left(1-\chi_{R}\right) F u\right) \\
=\left(u, \chi_{R} F u\right)+\underbrace{\int_{-\infty}^{-R}\langle u(x), F(x) u(x)\rangle_{\mathbb{C}^{2}} d x}_{I_{-}(R)}+\underbrace{\int_{R}^{+\infty}\langle u(x), F(x) u(x)\rangle_{\mathbb{C}^{2}} d x}_{I_{+}(R)} .
\end{gathered}
$$

Now,

$$
\begin{aligned}
& I_{-}(R)=-\underbrace{\left[\left\langle u(x), W_{-}(x ; R) u(x)\right\rangle_{\left.\mathbb{C}^{2}\right]_{-\infty}}^{-R}\right.}_{=0} \\
&+2 \int_{-\infty}^{-R} \operatorname{Re}\left\langle u^{\prime}(x), W_{-}(x ; R) u(x)\right\rangle_{\mathbb{C}^{2}} d x .
\end{aligned}
$$

It therefore follows that for $R>0$

$$
\left|\int_{-\infty}^{-R}\langle u(x), F(x) u(x)\rangle_{\mathbb{C}^{2}} d x\right| \leq \sup _{x \leq-R}\left\|W_{-}(\cdot, R)\right\|_{\mathbb{C}^{2} \rightarrow \mathbb{C}^{2}} \int_{-\infty}^{0}\left(|u|^{2}+\left|u^{\prime}\right|^{2}\right) d x
$$

and, analogously,

$$
\left|\int_{R}^{+\infty}\langle u(x), F(x) u(x)\rangle_{\mathbb{C}^{2}} d x\right| \leq \sup _{x \geq R}\left\|W_{+}(\cdot, R)\right\|_{\mathbb{C}^{2} \rightarrow \mathbb{C}^{2}} \int_{0}^{+\infty}\left(|u|^{2}+\left|u^{\prime}\right|^{2}\right) d x .
$$

Therefore we get

$$
\left|\left(u,\left(1-\chi_{R}\right) F u\right)\right| \leq 2 \alpha(R)\left(\|u\|_{L^{2}}^{2}+\left(u, \frac{1}{2} D_{x}^{2} u\right)\right),
$$

whence, picking $R_{0} \gg 1$ so that, say, $\alpha(R)<1 / 4$ for all $R \geq R_{0}$,

$$
\begin{aligned}
&(1-2 \alpha(R)) \frac{1}{2} D_{x}^{2}+\chi_{R} F-2 \alpha(R) \leq \frac{1}{2} D_{x}^{2}+F \\
& \leq(1+2 \alpha(R)) \frac{1}{2} D_{x}^{2}+\chi_{R} F+2 \alpha(R)
\end{aligned}
$$


on $C_{0}^{\infty}\left(\mathbb{R} ; \mathbb{C}^{2}\right)$, and also on $H^{2}\left(\mathbb{R} ; \mathbb{C}^{2}\right)$. Since $\chi_{R} F$ is $D_{x}^{2}$-compact for every $R>0$, we thus conclude by Proposition 2.3 that for all $R \geq R_{0}$

$$
\begin{aligned}
-2 \alpha(R)= & \inf \operatorname{Spec}_{\mathrm{ess}}\left((1-2 \alpha(R)) \frac{1}{2} D_{x}^{2}+\chi_{R} F-2 \alpha(R)\right) \\
& \leq \inf \operatorname{Spec}_{\mathrm{ess}}\left(\frac{1}{2} D_{x}^{2}+F\right) \\
& \leq \inf \operatorname{Spec}_{\mathrm{ess}}\left((1+2 \alpha(R)) \frac{1}{2} D_{x}^{2}+\chi_{R} F+2 \alpha(R)\right)=2 \alpha(R) .
\end{aligned}
$$

Hence, by taking the limit as $R \rightarrow+\infty$,

$$
0=\inf \operatorname{Spec}_{\mathrm{ess}}\left(\frac{1}{2} D_{x}^{2}+F\right) \in \operatorname{Spec}_{\mathrm{ess}}\left(\frac{1}{2} D_{x}^{2}+F\right) \subset[0,+\infty) .
$$

We have now to show that $[0,+\infty) \subset \operatorname{Spec}_{\text {ess }}\left(\frac{1}{2} D_{x}^{2}+F\right)$. Since 0 is in the essential spectrum, we may take a corresponding Weyl sequence $\left\{w_{k}\right\}_{k \geq 1} \subset$ $H^{2}\left(\mathbb{R} ; \mathbb{C}^{2}\right)$, that is, a sequence such that (w- $L^{2}$ stands for "weakly in $L^{2}$ ")

$$
\left\{\begin{array}{l}
\left\|w_{k}\right\|_{L^{2}}=1, \quad w_{k} \stackrel{\mathrm{w}-L^{2}}{\longrightarrow} 0, \\
\left\|\left(\frac{1}{2} D_{x}^{2}+F\right) w_{k}\right\|_{L^{2}} \longrightarrow 0,
\end{array} \text { as } k \rightarrow+\infty .\right.
$$

By (3.11) we get constants $C_{1}>0, C_{2} \in \mathbb{R}$ such that

$$
C_{1} D_{x}^{2} / 2+C_{2} \leq D_{x}^{2} / 2+F \text { on } H^{2}\left(\mathbb{R} ; \mathbb{C}^{2}\right),
$$

so that $\left\|D_{x} w_{k}\right\| \leq C_{3}$ for all $k \geq 1$. It therefore follows that we may choose a subsequence $\left\{w_{k_{j}}\right\}_{j \geq 1} \subset\left\{w_{k}\right\}_{k \geq 1}$ with $D_{x} w_{k_{j}} \stackrel{\text { w }-L^{2}}{\longrightarrow} w_{0}$ for some $w_{0} \in L^{2}$. On the other hand, $w_{k} \stackrel{\mathrm{w}-L^{2}}{\longrightarrow} 0$ implies that $w_{0}=0$. Next, as $\chi_{R} F$ is $D_{x^{-}}^{2}$ compact, by possibly passing to a subsequence (that we keep denoting by $w_{k_{j}}$ ) we have $\left(w_{k_{j}}, \chi_{R} F w_{k_{j}}\right) \rightarrow 0$ as $j \rightarrow+\infty$, so that from (3.11), (3.12) we obtain

$$
(1-2 \alpha(R)) \limsup _{j \rightarrow+\infty}\left\|D_{x} w_{k_{j}}\right\|_{L^{2}}^{2} \leq 2 \alpha(R) \longrightarrow 0, \text { as } R \rightarrow+\infty .
$$

Hence $\left\|D_{x} w_{k_{j}}\right\|_{L^{2}} \rightarrow 0$ as $j \rightarrow+\infty$. Now, for each $\gamma \geq 0$, put $u_{j}:=$ $e^{i \sqrt{2 \gamma} x} w_{k_{j}}$. Obviously, we have $u_{j} \in H^{2}\left(\mathbb{R} ; \mathbb{C}^{2}\right)$ and

$$
\left(\frac{1}{2} D_{x}^{2}+F\right) u_{j}=e^{i \sqrt{2 \gamma} x}\left(\frac{1}{2} D_{x}^{2}+F\right) w_{k_{j}}+\gamma e^{i \sqrt{2 \gamma} x} w_{k_{j}}+\sqrt{2 \gamma} e^{i \sqrt{2 \gamma} x} D_{x} w_{k_{j}} .
$$

Therefore $\left\|\left(D_{x}^{2} / 2+F-\gamma\right) u_{j}\right\|_{L^{2}} \longrightarrow 0$ as $j \rightarrow+\infty$. Since $\left\|u_{j}\right\|_{L^{2}}=1$ and $u_{j} \stackrel{\mathrm{w}-L^{2}}{\longrightarrow} 0$ as $j \rightarrow+\infty$, we thus have that $\gamma \in \operatorname{Spec}_{\mathrm{ess}}\left(D_{x}^{2} / 2+F\right)$, for all $\gamma \geq 0$. This concludes the proof of the proposition.

By Lemma 2.1 and Proposition 3.2 the proof of the theorem is now complete. 
Remark 3.3. The case $0<\alpha \beta<1$ seems to be of a very different nature, and to require another approach. We shall deal with this case in a subsequent paper.

\section{REFERENCES}

[1] S. Agmon. Lectures on exponential decay of solutions of second-order elliptic equations: bounds on eigenfunctions of N-body Schr??dinger operators. Mathematical Notes, 29. Princeton University Press, Princeton, NJ; University of Tokyo Press, Tokyo, 1982. $118 \mathrm{pp}$.

[2] M. Hirokawa. The Dicke-type crossings among eigenvalues of differential operators in a class of non-commutative oscillators. Indiana Univ. Math. J. 58 (2009), 1493-1535.

[3] F. Hiroshima and I. Sasaki. Multiplicity of the lowest eigenvalue of non-commutative harmonic oscillators. Preprint 2012. To appear in Kyushu J. Math.

[4] P. D. Hislop and I. M. Sigal. Introduction to Spectral Theory. With applications to Schrödinger operators. Applied Mathematical Sciences, 113. Springer-Verlag, New York, 1996. $\mathrm{x}+337 \mathrm{pp}$.

[5] L. Hörmander. Symplectic classification of quadratic forms, and general Mehler formulas. Math. Z. 219 (1995), 413-449.

[6] T. Ichinose and M. Wakayama. Zeta functions for the spectrum of the non-commutative harmonic oscillators. Communications in Mathematical Physics 258 (2005), 697-739.

[7] T. Ichinose and M. Wakayama. Special values of the spectral zeta function of the noncommutative harmonic oscillator and confluent Heun equations. Kyushu J. Math. 59 No. 1 (2005), 39-100.

[8] K. Kimoto and M. Wakayama. Spectrum of non-commutative harmonic oscillators and residual modular forms. Preprint MI 2012.

[9] N. Lerner. Metrics on the phase space and non-selfadjoint pseudo-differential operators. Pseudo-Differential Operators. Theory and Applications, 3. Birkhäuser Verlag, Basel, 2010. $x i i+397 \mathrm{pp}$.

[10] K. Nagatou, M. T. Nakao and M. Wakayama. Verified numerical computations for eigenvalues of non-commutative harmonic oscillators. Numerical Funct. Analysis and Opt. 23 (2002), 633-650.

[11] H. Ochiai. Non-commutative harmonic oscillators and Fuchsian ordinary differential operators. Comm. in Math. Phys. 217 (2001), 357-373.

[12] H. Ochiai. Non-commutative harmonic oscillators and the connection problem for the Heun differential equation. Letters in Math. Phys. 70 (2004), 133-139.

[13] A. Parmeggiani and M. Wakayama. Oscillator representations and systems of ordinary differential equations. Proceedings of the National Academy of Sciences U.S.A., 98 (2001), 26-30.

[14] A. Parmeggiani and M. Wakayama. Non-commutative harmonic oscillators-I, -II. Forum Mathematicum 14 (2002), 539-604 ibid. 669-690.

[15] A. Parmeggiani. On the spectrum and the lowest eigenvalue of certain noncommutative harmonic oscillators. Kyushu Journal of Mathematics 58 (2004), 277322.

[16] A. Parmeggiani. On the spectrum of certain noncommutative harmonic oscillators. Annali dell'Università di Ferrara 52 (2006), 431-456.

[17] A. Parmeggiani. On the spectrum of certain non-commutative harmonic oscillators and semiclassical analysis. Communications in Mathematical Physics 279 (2008), 285308.

[18] A. Parmeggiani. Spectral theory of non-commutative harmonic oscillators: an introduction. Lecture Notes in Mathematics, 1992. Springer-Verlag, Berlin, 2010. xii+254 pp. 
[19] I. Sasaki. Schrödinger operators with repidly oscillating potentials. Integr. Equ. Oper. Theory 58 (2007), 563-571.

[20] M. A. Shubin. Pseudodifferential operators and spectral theory. Second edition. Springer-Verlag, Berlin, 2001. xii+288 pp.

[21] M. Wakayama. On simplicity of the lowest eigenvalue of non-commutative harmonic oscillators. Preprint 2012.

Department of Mathematics, University of Bologna, Piazza di Porta S.DoNATO 5, 40126 BOLOGNA, ITALY

E-mail address: alberto.parmeggiani@unibo.it

Department of Mathematics, University of Bologna, Piazza di Porta S.DoNATO 5, 40126 BOLOGNA, ITALY

E-mail address: alberto.venni@unibo.it 